Revista Latinoamericana de la Papa 23 (1): 35 - 55, 2019

ISSN: 1853-4961

http://ojs.papaslatinas.org/index.php/rev-alap/index

\title{
La innovación inclusiva para desarrollar ventajas competitivas en cadenas de valor de la papa en la zona andina
}

\author{
A. Devaux $^{1 / *} ;$ M. Ordinola ${ }^{1}$
}

Recibido: 03/05/2018

Aceptado: $18 / 06 / 2018$

Accesible en línea: Junio de 2019

\section{Resumen}

A pesar del uso cada vez mayor de los sistemas de innovación y los enfoques de desarrollo en base a cadenas de valor para estimular el crecimiento de los ingresos rurales, la reducción de la pobreza y una mayor equidad de género existe poco conocimiento sistemático sobre cómo operacionalizar los enfoques de la cadena de valor en diferentes contextos, y cómo ampliar sus beneficios. Este artículo considera el desarrollo de la cadena de valor en términos competitivos, como un tipo de intervención que promueve el desarrollo de ventajas competitivas y contribuye a la reducción de la pobreza, al mejorar los vínculos entre los pequeños agricultores, comerciantes, procesadores y consumidores dentro de una cadena de valor. El Enfoque Participativo de Cadenas Productivas (EPCP) se describe como un enfoque flexible que involucra a actores de la cadena, investigadores y otros proveedores de servicios en un proceso colectivo que explora las oportunidades de negocios potenciales para el beneficio de los pequeños agricultores y otros actores de la cadena. Este enfoque fue desarrollado y aplicado por primera vez en el Perú, y luego, a través del intercambio de conocimientos, ha sido validado por el CIP y organizaciones locales en Bolivia y Ecuador en la zona Andina. Se describen las experiencias con la implementación y el ajuste del enfoque en estos contextos, así como las condiciones que han permitido la promoción de las innovaciones como base para desarrollar ventajas competitivas. El documento concluye con lecciones sobre la implementación que contribuyen a mejorar el diseño de las intervenciones y la promoción de enfoques de la cadena de valor en diferentes contextos.

Palabras clave adicionales: desarrollo de cadenas de valor, innovación, escalamiento, pequeños agricultores, biodiversidad, medios de vida rurales

\section{Inclusive innovation for ventage development in value chain of potato in the Andean}

\section{Summary}

Despite the increasing use of innovation systems and development approaches based on value chains to stimulate the growth of rural incomes, poverty reduction and greater gender equality there is little systematic knowledge about how to operationalize the value chain approaches in different contexts, and how to extend their benefits. This article considers the development of the value chain in competitive terms, as a type of intervention that promotes the development of competitive advantages and contributes to the reduction of poverty, by improving the links between small farmers, merchants, processors and

\footnotetext{
* Autor para correspondencia. E mail: a.devaux@cgiar.org y andrej.devaux@gmail.com

1 Centro Internacional de la Papa, Lima, Perú.
} 
consumers within of a value chain. Participatory Market Chain Approach (PMCA) is described as a flexible approach involving value chain actors researchers and other service providers in a collective process that explores the potential business opportunities for the benefit of small farmers and other actors in the chain. This approach was developed and applied first in Peru, and then, through knowledge exchange, it has been validated by CIP and local organizations in Bolivia and Ecuador in the Andean region. Experiences about its implementation and adjustment of the approach in these contexts are described as well as the conditions that have allowed the promotion of innovations as a basis to develop competitive advantages. The document concludes with lessons on implementation that contribute to improving the design of interventions and the promotion of value chain approaches in different contexts.

Additional key words: value chain development, innovation, scaling, smallholders, biodiversity, rural livelihoods.

\section{Introducción}

La agricultura está cambiando rápidamente en los países en desarrollo en respuesta a una variedad de factores, incluyendo las reformas institucionales y de políticas, las mejoras en la educación y la salud de los agricultores, nuevas oportunidades de ingreso y las inversiones en infraestructura rural. Existen señales claras de que las agroindustrias están teniendo un impacto global significativo en el desarrollo económico y la reducción de la pobreza, tanto en las comunidades urbanas como rurales (FAO, 2013). El acceso sostenible a los mercados posibilita que los agricultores pobres puedan aumentar los ingresos de su trabajo como una de las estrategias para reducir la pobreza (Wiggins et al. 2013,). Pero a menudo están en desventaja para producir y realizar transacciones en cadenas alimentarias de alta calidad (es decir, generar ventajas competitivas) debido a su acceso todavía limitado a los servicios, financieros y no financieros, y a su baja capacidad organizativa para la comercialización colectiva. Además, si se considera que los mercados tradicionales a los cuales se conectan normalmente se caracterizan muchas veces por la desconfianza, la incertidumbre y los altos costos de transacción, sus conexiones con el mercado son todavía informales. Esto es particularmente cierto para los cultivos perecibles, como la papa, que se cultivan en pequeñas fincas en zonas montañosas. Para que la investigación agrícola genere ventajas competitivas que beneficien a los productores de las zonas rurales, debe complementarse con otros esfuerzos que mejoren el entorno normativo, aliviar las limitaciones de recursos y crear capacidad local para responder a los desafíos y oportunidades tecnológicas y económicas cambiantes. A pesar de los riesgos asociados con los mercados de alto valor, los cambios del entorno del sector agrícola pueden contribuir en el desarrollo de mejores servicios de apoyo para los agricultores, tales como tecnología, extensión, seguros y apoyo financiero. Pequeños productores con acceso a servicios de apoyo técnico se han mostrado dispuestos a adoptar nuevas tecnologías e invertir para aprovechar las oportunidades de mercado (Royer et al. 2016).

En una recopilación de casos de investigación se presentan los resultados de trabajos recientes del consorcio 
CGIAR $^{2}$ y sus asociados en África, Asia y América Latina en los cuales se analizan las oportunidades que surgen de los mercados nuevos y en expansión de productos agrícolas, y se identifican los retos para la participación de los pequeños productores en esos mercados y los beneficios derivados de su participación (Devaux et al. 2016). En la zona Andina, la Iniciativa Papa Andina en los Andes utilizó la acción colectiva en dos enfoques para fomentar la innovación en la cadena de mercado: el Enfoque Participativo de Cadenas Productivas (EPCP) (Devaux et al. 2009) y las Plataformas de Innovación (Thiele et al. 2011b). Ambos enfoques buscan promover la interacción de los pequeños productores de papa con los actores del mercado y los proveedores de servicios agrícolas para favorecer alianzas y acuerdos contractuales en respuesta a nuevas oportunidades de mercado.

El presente artículo plantea algunas perspectivas sobre el desarrollo de la cadena de valor (DCV), y destaca las experiencias del Centro Internacional de la Papa (CIP) con el EPCP. Este enfoque, desarrollado originalmente para aumentar la competitividad y mejorar los medios de vida de los pequeños productores de papa en el Perú, también ha demostrado ser útil en otras cadenas de mercado y en otras partes de los Andes y del mundo. El artículo presenta las experiencias de implementación y los ajustes del enfoque en los Andes analizando los factores que han influido en su implementación y considera algunas lecciones surgidas de las experiencias en diferentes contextos para mejorar el diseño de las intervenciones usando el enfoque EPCP

\footnotetext{
2 el CGIAR es una asociación de investigación global para un futuro con seguridad alimentaria, http://www.cgiar.org/
}

como herramienta de investigación para el desarrollo y para analizar las condiciones de réplica y adaptación.

\section{Perspectivas sobre los conceptos de desarrollo de la cadena de valos}

Los conceptos de cadena de valor representan un cambio importante en el pensamiento sobre el desarrollo y las relaciones entre productores agrícolas, comerciantes, procesadores y consumidores. El término "cadena de valor" se utiliza de diferentes maneras en la literatura profesional. En este artículo, una cadena de valor se refiere al conjunto de actores y mercados relacionados entre sí que transforman insumos y servicios en productos con atributos que los consumidores están dispuestos a comprar. Millones de personas de bajos ingresos una gran parte mujeres - participan en cadenas de valor agrícola como productores, comerciantes, procesadores y minoristas. Muchos millones más, incluyendo a la mayoría de los pobres del mundo en desarrollo, participan en cadenas de valor agrícolas como trabajadores o consumidores. Por lo tanto, mejorar el desempeño de las cadenas de valor agropecuaria beneficiará a un gran número de personas (Reardon y Timmer 2012, Reardon et al., 2012).

El término "desarrollo de la cadena de valor" (que abreviaremos como DCV en el artículo) describe un tipo de intervención que apunta a abordar la pobreza a través de vínculos mejorados entre las empresas y los productores rurales. Ha sido definido como un "cambio positivo o deseable en una cadena de valor para extender o mejorar las operaciones productivas y generar beneficios sociales: generación de ingresos y empleo, crecimiento económico, desempeño ambiental, equidad de género y otros objetivos de 
desarrollo sostenible" (UNIDO 2011). Desde esta perspectiva, muchas agencias de desarrollo, donantes y gobiernos han adoptado el enfoque de cadena de valor para el desarrollo como un elemento clave de sus estrategias de reducción de la pobreza rural (Humphrey y NavasAlemán, 2010).

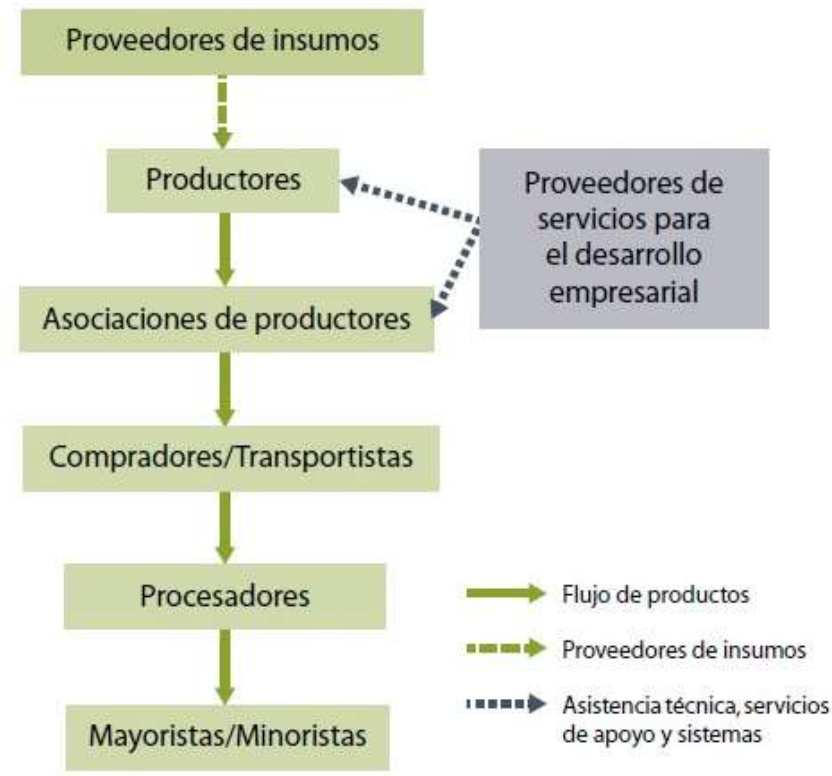

Figura 1. Cadena de valor estilizada.

Fuente: Devaux et al. 2016, figura 14.1

En contraste con los enfoques tradicionales de investigación y desarrollo (I+D) agrícola, que se centran en mejorar las capacidades de los pequeños productores para aumentar su productividad o administrar mejor los recursos naturales, el enfoque DCV desafía a las organizaciones de $\mathrm{I}+\mathrm{D}$ a trabajar con diversos actores para comprender el desempeño de la cadena de valor e identificar opciones mutuamente beneficiosas para mejorar el rendimiento y la eficiencia de la cadena, en base al desarrollo de innovaciones.

Si bien la globalización de los mercados ofrece oportunidades para comercializar productos de mayor valor que simplemente no existían antes, estos mercados generalmente demandan mucho más en términos de perspicacia empresarial, eficiencia y atención a las normas de calidad e inocuidad alimentaria que los mercados de productos tradicionales (Reardon et al. 2009). La participación en las cadenas de valor para mercados más exigentes requiere que los pequeños productores entreguen suministros regulares de productos de calidad consistente y en cantidad suficiente. El cumplimiento de estas condiciones requiere acceso a la tierra, insumos, tecnología, conocimiento, organización, capacidad, habilidad e infraestructura, que pueden no existir en algunas comunidades o hacer falta en algunos grupos de productores pobres. Se requieren también políticas públicas para adecuar las estrategias del gobierno a las 
diferentes situaciones para apoyar la participación de los pequeños productores en cadenas de valor más dinámicas. Los pequeños productores, en comparación con los agricultores medianos y grandes, están en desventaja en estas transformaciones, ya que muchas veces están ubicados en zonas con menos infraestructura privada y pública, están más alejados de los mercados, y tienen condiciones menos favorables para una agricultura de altos rendimientos y de alta calidad.

En este contexto, el CIP, en el marco de sus actividades en la zona andina, ha desarrollado un enfoque participativo más integrado, que combina la innovación agrícola, institucional y en el marco de las cadenas de valor, buscando un efecto sinérgico. Este enfoque, llamado Enfoque Participativo en Cadenas Productivas (EPCP), busca ser flexible e involucra a pequeños agricultores, agentes de mercado (comerciantes, empresas, procesadores, entre otros), investigadores y otros proveedores de servicios en un proceso colectivo que identifica y explora oportunidades de negocios potenciales que pueden beneficiar equitativamente a los diversos actores de una cadena seleccionada (Bernet et al. 2006). Fue desarrollado y aplicado por primera vez en los Andes, para aumentar la competitividad de la cadena del mercado de la papa, que constituye un componente importante del sistema agroalimentario local, y para contribuir a mejorar los medios de vida de los pequeños agricultores. El CIP ha encabezado el desarrollo del EPCP y ha apoyado el desarrollo de las capacidades locales necesarias para facilitar los procesos de innovación exitosos (Devaux et al. 2013).

\section{Desarrollo del EPCP y sus principales características}

Desde finales de 1990, la iniciativa regional Papa Andina ${ }^{3}$ ha trabajado para fortalecer la capacidad de las organizaciones de investigación y desarrollo en Bolivia, Ecuador y Perú con el objetivo general de aumentar la competitividad y mejorar los medios de vida de los pequeños productores de papa (Devaux et al., 2011). En el 2002, los científicos sociales del CIP, el programa Papa Andina y el Proyecto de Innovación y Competitividad de la Papa en Perú (Proyecto INCOPA) ${ }^{4}$ comenzaron a experimentar con un enfoque participativo conocido como Evaluación Rápida de Sistemas de Conocimiento Agrícola (RAAKS por sus siglas en inglés) (Engel \& Salomon, 1997), con la finalidad de estimular la innovación agrícola. Este enfoque reúne a diversas partes interesadas en un proceso participativo que estimula el aprendizaje colectivo, fomenta la confianza y promueve la innovación. Papa Andina empleó el RAAKS para explorar oportunidades de mercado involucrando a los pequeños agricultores, junto con actores de la cadena, investigadores y otros proveedores de servicios.

La participación de los comerciantes, supermercados, procesadores de alimentos $\mathrm{y}$ chefs en un proceso de investigación para el desarrollo, fue una

\footnotetext{
${ }^{3}$ El Programa Regional Papa Andina, implementado por el CIP con el apoyo de la Agencia Suiza para el Desarrollo y la Cooperación (COSUDE), ha trabajado desde 1999 hasta 2010 orientándose a fortalecer la capacidad de las organizaciones de Investigación y Desarrollo (I+D) de Bolivia, Ecuador y Perú, con el objetivo de aumentar la competitividad y mejorar los medios de vida de los pequeños productores de papa. 4 El Proyecto INCOPA, auspiciado por el Centro Internacional de la Papa (CIP) y con el apoyo de la Agencia Suiza para el Desarrollo y la Cooperación (COSUDE) fue formulado para mejorar la competitividad de la cadena de valor de la papa en Perú con énfasis en las papas nativas cultivadas por pequeños agricultores de las zonas alto-andinas.
} 
ruptura radical con los esfuerzos anteriores de I+D participativos, que se limitaban a investigadores y agricultores. Al implementarse las experiencias se añadieron pasos adicionales a RAAKS para el desarrollo de nuevos productos, y en ese contexto surgió un nuevo enfoque: el EPCP, que fue implementado en Perú y después validado en Bolivia en el 2003. En los años siguientes el enfoque fue aplicado en diferentes contextos y ampliamente documentado (Ordinola et al., 2009; Devaux et al., 2011).

El EPCP involucra a los actores que participan en una cadena de mercado, así como a los proveedores de servicios financieros y no financieros, públicos y privados, de apoyo a la cadena (tales como investigadores, proveedores de crédito y profesionales del desarrollo) en un proceso facilitado en el que se identifican y desarrollan innovaciones para aprovechar las oportunidades de mercado. Estas innovaciones pueden ser tecnológicas, comerciales e institucionales. La aplicación del EPCP está estructurada en tres fases que pueden durar alrededor de 12 a 18 meses según el contexto público, privado y los actores involucrados, como se presenta a continuación (Bernet et al., 2006; Antezana et al., 2008).
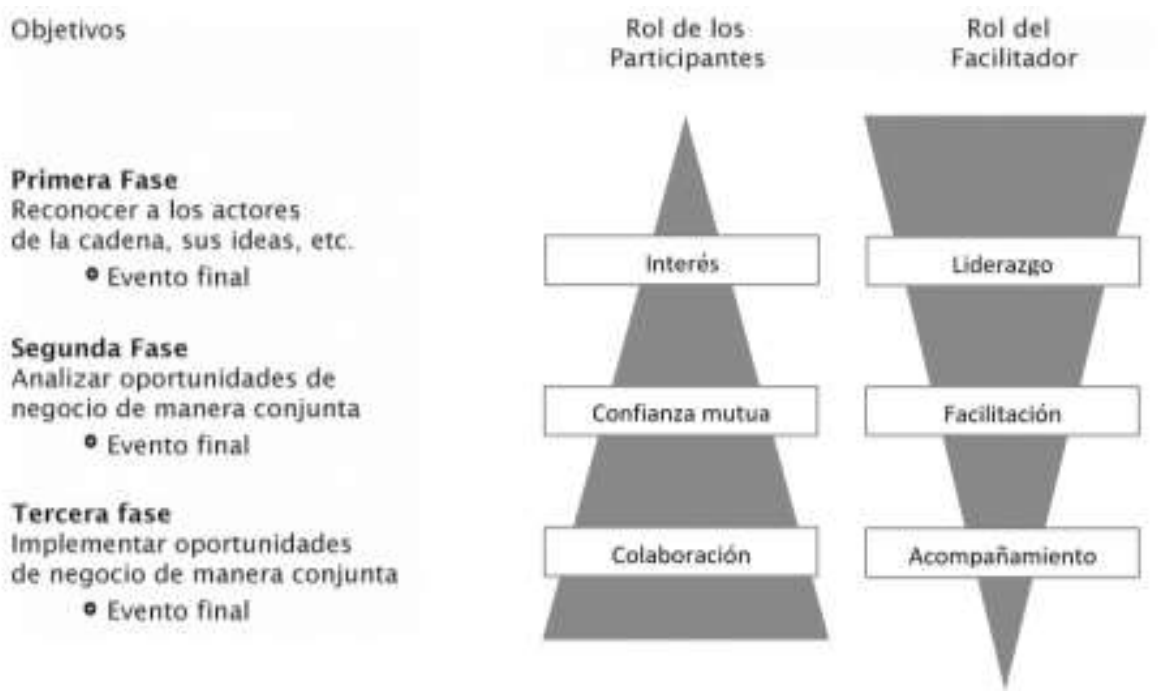

Figura 2. La estructura de la metodología en 3 fases del EPCP

Fuente: Tomado de Bernet, et al, 2006

FASE 1. Conocer los actores de la cadena de mercado y sus actividades mediante un diagnóstico. El EPCP es iniciado por una organización de I+D que lidera la selección de la cadena de mercado en la que se trabajará, identificando posibles socios de I+D y realizando investigaciones exploratorias y de diagnóstico participativo en la cadena. Esta fase, que puede durar de dos a cuatro meses, concluye con un evento público para discutir los resultados del diagnóstico, generar ideas para posibles innovaciones y motivar a los actores de la cadena de mercado y proveedores de servicios a participar en la Fase 2.

FASE 2. Análisis conjunto de oportunidades de mercado potenciales y de necesidades de innovación para 
aprovechar las oportunidades. Los representantes de las organizaciones de I+D participantes facilitan la exploración y el análisis de las oportunidades potenciales del mercado. Los actores de la cadena y los proveedores de servicios, organizados en grupos temáticos de interés, sostienen reuniones de trabajo para analizar las oportunidades haciendo uso de estudios técnicos o de mercado. Un segundo evento público se lleva a cabo al final de la Fase 2, para discutir las oportunidades de negocio priorizadas y para alentar a nuevos actores con el conocimiento adecuado y la experiencia complementaria a unirse a la Fase 3.

FASE 3. Desarrollo conjunto de innovaciones. Durante esta fase final, los grupos se enfocan en el desarrollo de productos y pruebas de mercado y en el lanzamiento de innovaciones específicas. En esta fase, las empresas juegan un rol más protagónico. Esto puede requerir trabajos especializados como pruebas de procesamiento, embalaje, etiquetado o marca. El ejercicio EPCP se cierra con un tercer evento público en el que las innovaciones de mercado desarrolladas se presentan a una amplia red de interesados como funcionarios públicos, donantes potenciales, responsables políticos, tomadores de decisiones del sector privado (supermercados, procesadores, chefs), público y representantes de los medios de comunicación.

Las experiencias descritas en este artículo ilustran ejemplos concretos de aplicación del enfoque de cadena y sus resultados en diferentes contextos.

\section{Experiencias en desarrollo de cadenas revisadas en la Zona Andina}

\begin{tabular}{lll}
\multicolumn{1}{c}{ Casos } & País & \multicolumn{1}{c}{ Fuentes principales } \\
\hline $\begin{array}{l}\text { (1) La revolución de la papa nativa en Perú } \\
\begin{array}{l}\text { (2) Accediendo a mercados de alta } \\
\text { diferenciación en Huancavelica, Perú }\end{array}\end{array}$ & Peru & $\begin{array}{l}\text { Ordinola } \text { et al. 2009, 2013 y 2014, } \\
\text { Horton y Samanamud, 2013 } \\
\text { Ordinola y Bellido, 2016 }\end{array}$ \\
$\begin{array}{l}\text { (3) Conservación y Comercialización de } \\
\text { papas nativas en Potosí, Bolivia }\end{array}$ & Bolivia & Horton et al. 2013c \\
$\begin{array}{l}\text { (4) La revalorización de las papas nativas en } \\
\text { el Ecuador }\end{array}$ & Ecuador & $\begin{array}{l}\text { Montesdeoca } \text { et al. 2013 y } \\
\text { Montesdeoca, 2016 }\end{array}$
\end{tabular}

\section{La revolución de la papa nativa en el Perú}

De 2001 a 2010, más de 20 organizaciones públicas, ONG y empresas trabajaron el Proyecto Innovación y Competitividad de la Papa en Perú (INCOPA) en el marco de la Iniciativa Regional Papa Andina, con el objetivo de promover innovaciones en la producción y comercialización de la papa nativa y mejorar la competitividad de esta cadena en beneficio de los pequeños agricultores que la producían. Con este fin, se desarrolló y usó el enfoque de investigación y desarrollo aplicado, denominado EPCP. INCOPA trabajó para 
vincular a los pequeños productores de papas nativas con investigadores, profesionales del desarrollo y una gama de actores de la cadena del mercado de la papa (supermercados, procesadores, chefs) para capitalizar la biodiversidad de las papas nativas $y$ sus atributos culinarios, nutricionales y culturales. La implementación del EPCP fue complementado con esfuerzos para promover las alianzas entre actores de la cadena mediante plataformas de innovación, para aumentar la conciencia pública sobre las virtudes de las papas nativas y apoyar la formulación de políticas en apoyo a estas cadenas. El EPCP actuó como un detonador de innovaciones que, en combinación con diferentes intervenciones en el ámbito técnico, institucional y político, desencadenó procesos de innovación comerciales, productivas e institucionales que siguen hasta hoy y contribuyeron a lo que se puede llamar "la revolución de la papa nativa en el Perú" (Horton y Samanamud, 2013).

En el Perú se implementaron dos ciclos del EPCP. La primera aplicación se centró en la comercialización de variedades mejoradas de papa; el segundo ciclo se enfocó en la comercialización de papas nativas. Los participantes en los ejercicios fueron no sólo investigadores, funcionarios del ministerio de agricultura y actores típicos de la cadena de mercado, sino también chefs y gerentes de supermercados. Esta fue la primera vez que un grupo tan diverso se reunía para colaborar en opciones para mejorar y promover la papa en el Perú. Los "nuevos actores" trajeron energía renovada y nuevas ideas a discusiones que antes habían estado dominadas por profesionales de I+D.

Las aplicaciones del EPCP dieron lugar a dos nuevos productos elaborados con papas nativas de Perú. T'ikapapa fue lanzada como la primera marca de papa nativa fresca gourmet vendida en un supermercado de la capital peruana. Poco después, Jalca Chips, un producto innovador de chips de papa de diferentes colores naturales (azul, roja, amarilla) también fue producido a partir de papas nativas. Ambos productos estaban disponibles en las tiendas de comestibles $\mathrm{y}$ fueron reemplazados poco a poco por innovaciones comerciales mejoradas, en un proceso de imitación creativa. Hoy en día han aparecido en el mercado más de 20 productos similares, algunos de los cuales se exportan, como se ilustra en la figura 3 (Ordinola et al., 2009).

Además de los nuevos productos, surgieron ideas importantes para iniciativas políticas y campañas de sensibilización y promoción de la papa y su consumo durante el segundo ciclo del EPCP. Quizá lo más notable fue el Día Nacional de la Papa del Perú. Se estableció en 2005 y se ha celebrado anualmente desde entonces tanto en Lima como en las diferentes regiones. El Ministro de Agricultura estableció una comisión especial para organizar este evento, incluyendo participantes del ejercicio del EPCP. Hoy en día esta comisión continúa funcionando como una red institucionalizada y el MINAGRI asigna un presupuesto anual a esta celebración que promueve cada año el sector de la papa en la sociedad peruana. Esta comisión apoyó los esfuerzos de los funcionarios del gobierno peruano en su solicitud para que las Naciones Unidas declarase el 2008 como Año Internacional de la Papa. Cuando esta propuesta fue aceptada, la comisión encabezó numerosas actividades de divulgación en el Perú para esta celebración que se extendió a lo largo del año. La combinación de innovaciones en 
la cadena de mercado, los cambios en las políticas para promover la modernización del sector comercial y la conciencia pública han contribuido a cambiar la imagen y percepción de las papas nativas en el Perú.

El proyecto INCOPA y su red de colaboradores público-privados respaldaron las iniciativas de los pequeños empresarios más innovadores. Estos esfuerzos fueron apoyados por ONGs que trabajaban en los territorios donde se producían estas papas, para ayudar a los productores a organizarse y poder abastecer con productos de calidad según los requerimientos del mercado. La plataforma CAPAC Perú (Cadenas Productivas Agrícolas de Calidad en Perú), que surgió como una innovación institucional y a partir del EPCP, ha apoyado la cadena de valor de la papa nativa promoviendo la interacción entre los productores y los agentes de mercado
(Ordinola et al., 2009). La participación de diferentes actores del mercado, incitada por los resultados prometedores que observaron con las pequeñas empresas, llevó a la creación de una serie de nuevos productos a base de papa y de diferentes marcas de chips de papas nativas de calidad superior, que hizo aparecer en el mercado una gama de productos promovidos por multinacionales como Frito Lay y Gloria y también por pequeñas empresas de provincias, para responder a demandas diversas del mercado. El EPCP suscitó un proceso de "gatillo" (disparador), donde la segunda y tercera generación de innovaciones resultaron, muchas veces, más importantes que las primeras que fueron desarrolladas durante su aplicación inicial (Ordinola et al., 2013; Proexpansión, 2011). La figura 3 ilustra este proceso. 


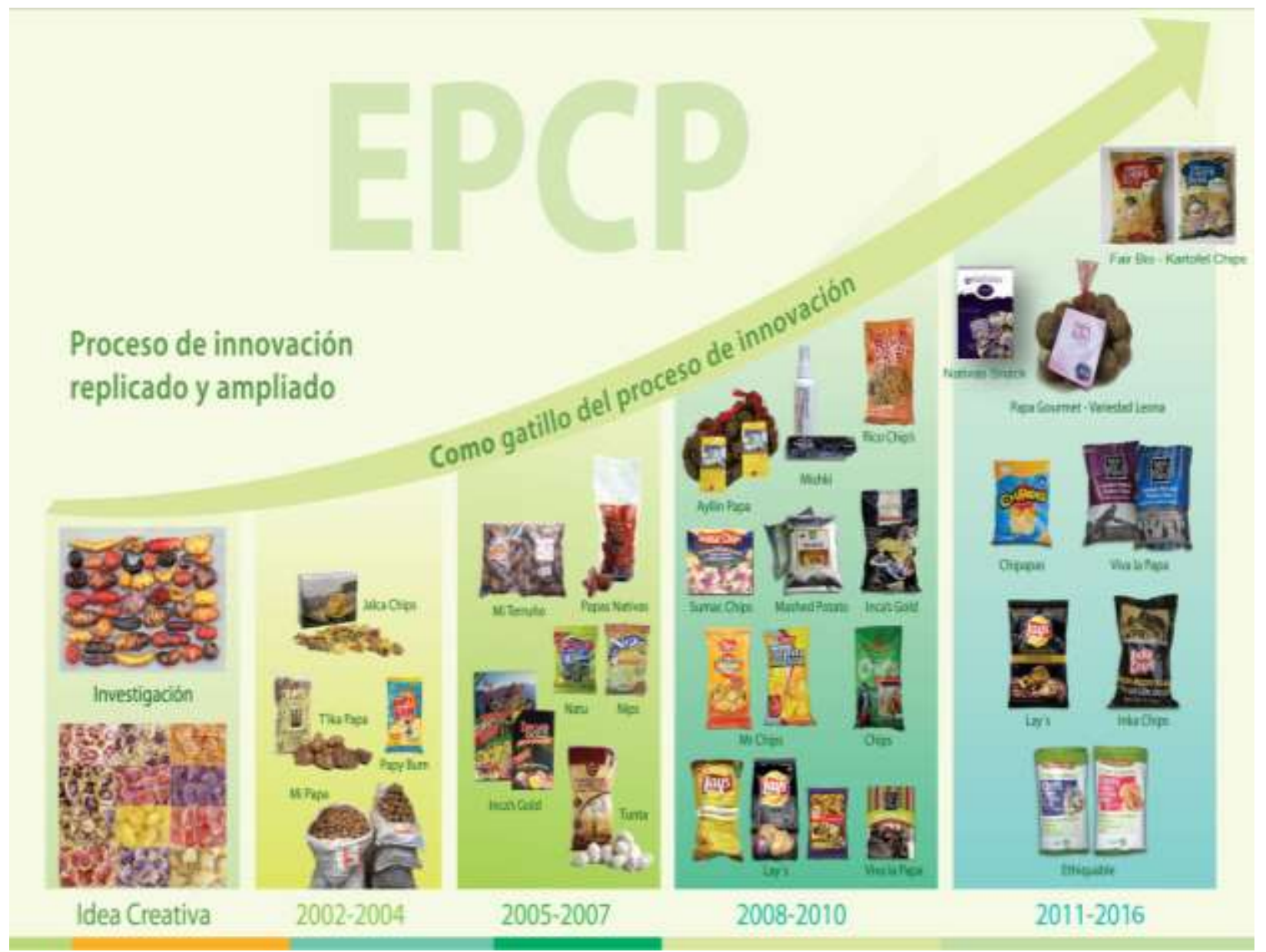

Figura 3 El EPCP como un detonador de la innovación para el desarrollo de nuevos productos de papa en el Perú

Fuente: Actualizado en base a Ordinola, et al, 2009

En la provincia de Andahuaylas, Región Apurímac, Perú, se implementó un estudio de impacto para evaluar los alcances e impactos iniciales de la intervención del Proyecto INCOPA a través de la plataforma institucional CAPAC Perú ${ }^{2}$, utilizándose el marco metodológico de evaluación "vías de impacto" (Maldonado et al., 2011). En esa región, el proyecto INCOPA

${ }^{5}$ Cadenas Productivas Agrícolas de Calidad en el Perú, (CAPAC Perú) era una organización de promoción social, económica y tecnológica de segundo nivel, orientada a brindar servicios especializados en el desarrollo de las cadenas productivas de papa y otros tubérculos que se cultivan en el Perú. promovió el uso de la biodiversidad y el desarrollo de oportunidades de mercado para las papas nativas para mejorar la competitividad de la cadena de la papa utilizando el EPCP. Para este estudio, se elaboró una lista de 80 productores en la zona de intervención utilizando el criterio de estratificación por ubicación geográfica (las unidades de muestreo se agrupan según su ubicación geográfica). Asimismo, se definió como grupo contrafactual a 66 agricultores de las mismas zonas. 
Entre las principales conclusiones de este estudio de caso se destacó que:

1. los productores de papa de la zona estudiada han ampliado y diversificado sus relaciones comerciales, principalmente por la mayor demanda de la papa nativa tanto a nivel local como de los supermercados y la agroindustria en Lima;

2. Los pequeños productores de la zona estudiada han logrado desarrollar habilidades de gestión comercial y han mejorado sus técnicas de postcosecha de acuerdo con la mayor demanda de los nuevos mercados a los cuáles han accedido;

3. Los productores de papa (grupo objetivo) pertenecen a organizaciones que mantienen relaciones y vínculos con otras instituciones proveedoras de servicios, lo cual les permite acceder a asistencia técnica y al desarrollo de una gestión comercial más eficiente;

4. Los productores involucrados en el proyecto obtienen mayores ingresos promedios a través de mejores precios (26 por ciento por encima del grupo control) y mayores volúmenes de ventas de papa, especialmente de papa nativa.

Posteriormente, en un estudio de caso sobre la participación en cadenas de valor de papas nativa de los productores de estas papas en la sierra central del Perú, Dan Tobin (2016) investigó la diferenciación social dentro de comunidades entre participantes y no participantes, así como las razones para la inclusión o exclusión de los hogares en la articulación a la cadena. Estos hallazgos indican que la participación en cadenas de valor no es necesariamente beneficiosa para todos los pequeños productores. Para que dichos programas tengan amplios beneficios sociales se necesitan políticas $\mathrm{y}$ otros arreglos institucionales para analizar y minimizar los riesgos de participación en tales cadenas de valor y para brindar apoyo para la participación en otros tipos de mercados menos exigentes. Dado que los mercados de alto valor a menudo requieren una mayor inversión y activos, probablemente no exista una combinación adecuada entre los hogares de más bajos recursos y los mercados de alto valor. Horton y Samanamud (2013), indican también que las familias con menor extensión de tierras, menor educación, escaso acceso al crédito y redes sociales menos desarrolladas se han beneficiado menos de las nuevas oportunidades de mercado. Pero a pesar de estas consideraciones, estos mismos autores mencionan que proceso de innovación generado por el EPCP ayudó a mejorar la imagen de las papas nativas y a vincular a los pequeños productores con más capacidades a los mercados urbanos dinámicos para productos a base de papa.

Analizando el caso del desarrollo de las cadenas de papas nativas en Perú, el contexto económico y político del país ha sido favorable, incluyendo el apoyo del gobierno con políticas favorables, la participación del sector privado comprometido, el desarrollo del sector gastronómico que promueve productos nativos, y la integración en proyectos de la cooperación internacional, en este caso de COSUDE, que fortaleció el proceso de innovación mediante la capacitación y el acompañamiento a la implementación del EPCP y al desarrollo de las innovaciones. Este proceso ha posibilitado cambiar la situación anterior, que consideraba las papas nativas como alimento básico de subsistencia para los agricultores pobres de las alturas, con poco futuro en la 
agricultura orientada al mercado, y que ahora son reconocidas como un producto peruano especial y nutritivo que merece un premio (vía precio) en los mercados urbanos y restaurantes gourmet.

\section{Accediendo a mercados de alta diferenciación ${ }^{6}$ en Huancavelica Peru}

El caso de las papas nativas de Huancavelica (Perú) es interesante mencionar. La experiencia se ha orientado a la promoción productiva y comercial de las papas nativas para mercados de alta segmentación (comercio justo y productos orgánicos) tanto nacional como internacional. Como en el caso de experiencias con el EPCP en Bolivia, las innovaciones de mercado con las papas nativas que se desarrollaron anteriormente con el EPCP en Perú, sirvieron de insumos iniciales para desarrollar esta experiencia. Se ha logrado desarrollar un modelo innovador para el manejo y gestión sostenible de las acciones, impulsando la articulación de sectores públicos y privados en torno a la cadena de papas nativas $y$ en base a tres componentes.

Esta iniciativa combina (1) un componente relacionado con el mercado (el cual es el motor del modelo), donde se complementan las

\footnotetext{
6 En base a "Desarrollando la producción de la sierra en función a mercados de alta segmentación: El caso de las papas nativas de Huancavelica". Informe de consultoría preparado por Miguel Ordinola y Francisco Bellido. Setiembre 2016. Este proyecto lo implementó la institución CEDINCO con el apoyo financiero del Fondo Ítalo Peruano (FIP), con familias de diversas comunidades de Castrovirreyna, Huancavelica y Tayacaja y en alianza con la ENIEX Agrónomos y Veterinarios Sin Fronteras AVSF.
}

alternativas de los mercados nacionales e internacionales, asociados con productos de alta diferenciación comercial. Las intervenciones a este nivel tienen que ver con: i) identificación y relacionamiento comercial con mercados de alta segmentación principalmente internacionales (orgánico y comercio justo); ii) acceso a certificaciones de reconocido valor comercial (comercio justo y producción orgánica); iii) promoción comercial de productos diversificados; iv) acceso a mercados y estrategia de promoción comercial. (2) Un componente relacionado con la oferta de calidad y abastecimiento continúo para la consolidación del negocio en los diferentes mercados. De acuerdo a los mercados identificados se desarrolló la oferta en función a los estándares requeridos por la demanda. Para esto se implementaron las siguientes intervenciones: i) promoción de producción orgánica con un enfoque de demanda; ii) articulación con la investigación a nivel local (tesis); iii) conservación de la biodiversidad (trabajo con agricultores conservacionistas; iv) posibilitar el abastecimiento continuo del producto (dos campañas al año con pequeños sistemas de riego; v) manejo, producción y uso de semilla de calidad; (3) Un componente relacionado con los aspectos organizacionales, en sentido que la asociatividad es clave para generar escalas en la producción y desarrollar valor agregado. La lógica se orienta a la articulación de organizaciones (de tres provincias) con un trabajo de fortalecimiento organizacional para mejorar la eficiencia en la gestión. Las intervenciones tienen que ver con lo siguiente: i) promover un modelo empresarial; ii) acciones de fortalecimiento organizacional; iii) fortalecimiento de capacidades. 
Estas intervenciones se han trabajado inicialmente con 230 familias y han permitido: i) mejoras en los precios ya que se accede a mercados que reconocen (vía precios, el algunos casos los incrementos en precios han llegado al $100 \%$ ) la calidad de los productos diferenciados; ii) una mayor producción (cantidad) y una mejora sustancial de la calidad, ya que se promueve el acceso a las diferentes certificaciones requeridas por los mercados finales (ya están en su sexto año de abastecimiento); iii) desarrollar mayores capacidades de negociación en los diferentes mercados y se expresa en acceder a mejores condiciones de venta (formas de pago) y precios diferenciados (sobre todo en el segmento de comercio justo).

Se pueden rescatar aprendizajes de este caso:

Las papas nativas orgánicas acceden a un mercado de nichos específicos de alta calidad, en base a la eficiencia de su producción

En esta experiencia se ha podido identificar de manera directa el mercado de los productos a trabajar (internacionales y nacionales) y se ha desarrollado una respuesta adecuada y efectiva. El modelo nace a partir de la existencia de una demanda no satisfecha por productos orgánicos y el acceso a los mercados de Europa, que se han complementado con los nichos de demanda del mercado local.

El co-financiamiento es una parte de la estrategia para comprometer a los productores en la ejecución de proyectos

Una contrapartida tangible (monetaria o no monetaria) hace posible que los beneficiarios jueguen un rol activo en el trabajo. Los productores organizados (ahora en el marco de la Cooperativa Agropia) se han comprometido en el cofinanciamiento de las diversas intervenciones (por ejemplo, los pequeños sistemas de riego).

La provisión de servicios especializados es clave para el funcionamiento de los negocios

En esta experiencia los temas relacionados a manejo de producción orgánica, manejo de semilla de calidad, manejo de cultivo, manejo del agua, comercialización, organización y gestión empresarial, han sido reforzados en función a la obtención de una producción de calidad para responder adecuadamente al mercado.

Generación de alianzas públicoprivadas como elemento necesario para evitar intervenciones aisladas

Varias instituciones han participado de esta experiencia, empresas (nacionales e internacionales), gobiernos locales, instituciones públicas (por ejemplo, el MINAGRI), entre otras instituciones que han potenciado la experiencia. Las alianzas proporcionan solidez y estabilidad a los diferentes servicios. La generación de confianza es clave para optimizar el trabajo entre los diferentes actores de los negocios.

La promoción de la demanda por parte de la empresa privada como sostén de mediano plazo.

Esto ha sido el caso de los mercados externos a los cuáles han accedido los pequeños productores que participan en esta experiencia (Francia, Bélgica, Alemania), supermercados a nivel local (Wong-CENCOSUD) y tiendas especializadas de productos agroecológicos y orgánicos. 


\section{Conservación y Comercialización de papas nativas en Potosí, Bolivia}

Desde 2007 al 2010, el proyecto Alianza Cambio Andino ${ }^{7}$ analizó los procesos y productos de implementación del EPCP en estudios de casos en diferentes cadenas en los Andes. En este artículo, presentamos el caso de las papas nativas en el norte de Potosí, Bolivia. PROINPA y el Centro de Desarrollo Agrícola (CAD) han trabajado durante varios años para conservar la biodiversidad de la papa y otros cultivos andinos y para reducir la pobreza rural en el norte de Potosí. La región en su conjunto produce un pequeño excedente de papas en el mercado, que varía ampliamente de un año a otro dependiendo de las condiciones climáticas. La agricultura genera pocos ingresos en efectivo, y los jóvenes abandonan el campo en busca de empleo en minas y ciudades. Esta aplicación del EPCP busco aprovechar la demanda creciente de papas nativas en mercados urbanos.

La organización CAAD y la organización de agricultores PRONAPA desarrollaron un nuevo producto de papa nativa denominado " Miskipapa", que consistía en papas nativas seleccionadas y lavadas que se vendían en mallas. Ha sido comercializado con el apoyo de CAD durante 3 años en supermercados en La Paz y Cochabamba, en la tienda de un sindicato minero, en dos hoteles turísticos y en mercados de agricultores. Debido a las limitaciones tanto en la oferta como en la demanda de papas nativas, los beneficios económicos para los

\footnotetext{
${ }^{7}$ La Alianza Cambio Andino es un programa regional de cooperación entre organizaciones sociales y de negocios de Bolivia, Colombia, Ecuador y Perú, con el fin de incluir métodos participativos para la inclusión de los pequeños productores rurales en la innovación agropecuaria.
}

agricultores fueron reducidos. Una vez que terminó el apoyo en el marco del EPCP, la organización dejó de operar en la región y la asociación de agricultores se disolvió. Miskipapa desapareció del mercado. Muchos de los líderes productores que tuvieron un rol importante en el proceso han migrado. Por otro lado, las condiciones del modelo económico del gobierno, las condiciones agroclimáticas del lugar y la situación de pobreza de los productos no favorecieron el éxito de la implementación del EPCP.

Actualmente, existen algunas iniciativas privadas (ONG, fundaciones, empresas) vinculadas a la producción y comercialización de papas nativas, que podrían tomarse como efectos indirectos o imitaciones creativas de los resultados de los primeros esfuerzos con el EPCP para promover las papas nativas en mercado boliviano. Estos fueron promovidos por la Fundación PROINPA luego de haber participado en la iniciativa inicial de EPCP con papas nativas. La compañía de procesamiento Andina de Papa (Pa\&Pa) ubicada en Cochabamba proporciona un bocadillo de papa nativa en forma de hojuelas de la variedad de color Pinta Boca a la compañía boliviana de aviación - BoA, la aerolínea de bandera boliviana, propiedad del Estado. El proyecto inicial (2012) consideró la industrialización de cuatro variedades de papas nativas promovidas por la Fundación PROINPA, Pa\&Pa y productores del municipio de Colomi (Cochabamba). Otra experiencia que se puede considerar como una imitación creativa en Cochabamba, es la iniciativa que promovió la papa nativa "Pinta Boca" en los mercados urbanos bajo la marca RICA PAPA. Se comercializó como un producto en fresco de papa gourmet en los supermercados. Es un negocio familiar que comenzó a recolectar 
variedades nativas de papa para abastecer a los supermercados en La Paz, Cochabamba, Oruro y Santa Cruz, además de proporcionar el Programa Público de subsidio prenatal. Actualmente, el volumen de ventas mensuales de papas nativas es de aproximadamente cinco toneladas por mes.

Las políticas en favor del mercado de Perú proporcionaron un entorno más favorable para el uso del EPCP que las políticas del gobierno Boliviano, que enfatizaban más el papel del estado. Los entornos agroecológicos tienen también un efecto sobre los procesos de implementación y los resultados. En el altiplano boliviano, donde se hicieron las primeras experiencias con el EPCP y donde la pobreza es muy elevada limitando el acceso a servicios, las condiciones de producción son adversas debido al uso reducido de insumos y a los riesgos climáticos. Son limitantes severas para la implementación de los enfoques de desarrollo de la cadena de mercados agrícolas para la reducción de la pobreza rural. Las siguientes experiencias más exitosas se realizaron en zonas de producción en Cochabamba en condiciones de producción y socioeconómicas más favorables.

\section{La revalorización de las papas nativas en el Ecuador}

En noviembre de 2008, mediante la colaboración entre el CIP, a través del proyecto Alianza Cambio Andino, el Programa Papa del Instituto de Investigaciones Agrícolas y Pecuarias (INIAP), la ONG local Fundación Marco, y la asociación de productores del Consorcio de Productores de Papa (CONPAPA), se inició la aplicación del EPCP en la cadena de papas nativas de
Ecuador, para capitalizar la gran riqueza de variedades nativas de papa del país.

El diagnóstico cualitativo de la primera fase del EPCP involucró a 29 chefs y administradores de restaurantes y hoteles de diversas provincias del país para determinar el conocimiento y las actitudes existentes hacia las papas nativas $\mathrm{e}$ identificar nichos de mercado. Los resultados mostraron que en los restaurantes y en los hoteles de las ciudades se desconocían estas variedades y que no se las podía encontrar en los mercados. Sin embargo, los participantes coincidieron en afirmar que representaban un interesante potencial de negocio. Un año después de haber aplicado las 3 fases del EPCP, se lanzó la marca "papa nativa" en un Gran Evento Final del proceso EPCP con el slogan "descubriendo el sabor andino". El producto consistía en una malla de papas nativas seleccionadas y lavadas (Montesdeoca et al., 2013). El lanzamiento fue apoyado con ruedas de prensa y ferias gastronómicas en el marco de la celebración del Día Nacional de la Papa. Desarrollar el producto como una innovación comercial desencadenó innovaciones tecnológicas a nivel de la producción (fertilización, manejo de semillas, manejo de plagas) y en la presentación comercial de la papa como producto seleccionado y con nombre de marca. Para la asociación de productores CONPAPA significó elevar sus capacidades asociativas para el acopio y comercialización, que por primera vez comercializaba papas nativas en diversas presentaciones y mediante la articulación comercial con consumidores intermedios urbanos.

$\mathrm{Si}$ bien la marca "Papas Nativas Descubriendo el Sabor Andino" no prosperó en el mercado por problemas en el abastecimiento, altos costos de 
transacción y limitado volumen de demanda, los resultados del EPCP fueron importantes para poner "en vitrina" el valor de la papa nativa en mercados distintos a los del consumo tradicional y familiar local. Estos resultados fueron captados positivamente por la empresa INALPROCES, que por varios años apostaba por productos de alta calidad con criterios de responsabilidad social. INALPROCES, inspirada por la experiencia de la Iniciativa Papa Andina del CIP sobre comercialización de papas nativas en Perú, vio en el mercado ecuatoriano y en el extranjero, interesantes posibilidades de negocio a través de la producción y venta de hojuelas fritas de color obtenidas a partir de variedades nativas.

INALPROCES, en alianza con el INIAP, la Fundación Minga para la Acción Rural y la Cooperación, y el CIP en Ecuador, trabajaron en la identificación de papas nativas adecuadas para ser producidas por agricultores del CONPAPA y abastecer la demanda de procesamiento (Montesdeoca et al., 2013). Se seleccionaron 2 variedades de papa de color para producir hojuelas de calidad: Puca Shungo (Corazón Rojo) y Yana Shungo (Corazón Negro). Su característica principal es la coloración intensa en tonos rojizos y morados que las hacen ricas en antioxidantes, vitaminas y proteínas y las diferencian de las papas tradicionales. En el año 2011 salió al mercado la marca de hojuelas de color, KIWA, promovida con apoyo de la cooperación internacional como un modelo de negocio de responsabilidad social empresarial. Este modelo de negocio incluía el trabajo en colaboración con una serie de otros actores locales para mantener niveles de producción consistentes y para asegurar el suministro de semillas de calidad. El modelo también incluía servicios de capacitación para los agricultores, basado en la metodología de Escuelas de Campo de Agricultores. El modelo de negocio promovido por INALPROCES tuvo tanto éxito en sus inicios que, en 2011, su trabajo con los agricultores de papas andinas fue condecorado como el mejor proyecto de Responsabilidad Social Corporativa de Ecuador por la Cámara de Industria y Comercio EcuatorianoAlemana.

Desde su lanzamiento al mercado, el producto ha elevado sus ventas en el mercado nacional y en el mercado de exportación en distintas plazas de Estados Unidos, Europa y el Medio Oriente. Este crecimiento ha promovido la colaboración entre el CIP, el INIAP y los productores de CONPAPA, para llevar adelante acciones destinadas a potenciar la producción de semilla de calidad de las variedades Puca Shungo y Yana Shungo, seleccionar nuevas variedades, incrementar los volúmenes de su producción y planificar la compra y venta entre CONPAPA e INALPROCES.

El desarrollo de los chips de papas nativas Kiwa, es uno de los ejemplos de la evolución del proceso de innovación que, como observamos, continúa y evoluciona mucho después de que el EPCP haya finalizado formalmente. Esta innovación comercial ha requerido también innovaciones tecnológicas para responder a demandas del mercado. Por ejemplo, para satisfacer la demanda de semilla de las variedades utilizadas en la producción de hojuelas de Kiwa, se incluyeron estas variedades en el programa de multiplicación de semillas del invernadero de producción de semilla de alta calidad del INIAP. El caso de del producto KIWA es un ejemplo de cómo las oportunidades de mercado y el desarrollo de negocios con responsabilidad social, funcionan como 
fuerza impulsora para la formación y operación de alianzas público-privadas para el desarrollo rural. También existen otros retos a nivel productivo, principalmente con los cambios en el clima y las heladas en la Cordillera de los Andes ecuatorianos que afectó la producción hasta un $80 \%$ en el 2014. Se requiere seguir la selección de variedades que permitan un procesamiento de calidad. En el campo del procesamiento es necesario encontrar más opciones para usar estas papas, como el puré de papas y las papas fritas congeladas de papas nativas andinas para la exportación (Martin Acosta, CEO KIWA comunicación personal).

Observamos que en el contexto macro ecuatoriano las políticas en favor del mercado no eran tan favorables como en el caso peruano. Uno de los grandes retos ha sido el incentivo al emprendimiento. A pesar de que Ecuador es uno de los países con los mayores registros de marcas e invenciones en la zona andina, el desarrollo de dichas propuestas pocas veces se convierte en negocios, se crean pocas empresas y en mucha menor medida se generan oportunidades de empleo (Wong y Padilla, 2017). Del lado de los productores se requería un sistema general de apoyo a la inversión para expandir su producción. Ninguno de los agricultores tenía acceso al crédito, más bien cuando era necesario invertían sus ahorros familiares o pedían préstamos personales.

Con la participación de INALPROCESS, el producto basado en hojuelas de papas de color se ha desarrollado y expandido con presencia en los mercados nacionales e internacionales. En 2018, el producto de papa procesada representaba el $20 \%$ de las ventas de la empresa. La compañía planea expandirse principalmente en EE. UU., Medio Oriente y Europa del Este e ingresar al mercado con certificación orgánica (Martin Acosta, CEO de KIWA, comunicación personal)

\section{Lecciones para el desarrollo de cadenas de valor en diferentes contextos}

Las experiencias con el EPCP e intervenciones en cadenas de valor en diferentes contextos permiten identificar algunas lecciones que contribuyen a mejorar el diseño de las intervenciones para el desarrollo de cadenas de valor promoviendo la investigación y la innovación colectiva.

Un elemento importante en la promoción del EPCP es la integración siempre creciente del sector agropecuario en los mercados que incrementa las oportunidades de mercado para los pequeños agricultores. Desde la situación tradicional de vender directamente a los consumidores rurales o al mercado local, las cadenas de valor modernas han abierto las puertas a otros compradores: comerciantes de mercados rurales y urbanos, empresas procesadores, comercio justo y supermercados. La implementación del EPCP, mediante la interacción de los productores con actores de la cadena y el análisis de mercados, permite identificar nichos de mercado $\mathrm{y}$ nuevas oportunidades comerciales donde los pequeños productores pueden desarrollar una ventaja competitiva a partir de contar con una ventaja comparativa, en este caso la biodiversidad. En la guía del usuario del EPCP (Bernet et al., 2006), hay una herramienta llamada "filtro de impacto" que proporciona una evaluación cualitativa rápida del impacto esperado de diferentes oportunidades de mercado en favor de los pequeños productores considerando también los objetivos sociales y ambientales. Esta herramienta 
permite a las organizaciones de $\mathrm{I}+\mathrm{D}$ planificar y guiar las intervenciones de manera más efectiva.

Un enfoque holístico como el EPCP representa una nueva forma de hacer I+D agrícola. En lugar de emprender investigaciones y luego intentar transferir los resultados a los agricultores, el enfoque congrega toda una gama de actores relevantes -incluyendo agricultores, agentes del mercado, procesadores, investigadores y proveedores de servicios - para establecer prioridades y desarrollar conjuntamente innovaciones que respondan mejor a las demandas de la cadena de valor. El enfoque para el desarrollo de cadenas de valor nos lleva a destacar la importancia que hay que dar en los debates sobre seguridad alimentaria a los componentes de postcosecha y de cadena de valor en contraste con los apoyos dirigidos solo al sector productivo. Por lo tanto, para promover la seguridad alimentaria, la eficiencia de la cadena y el manejo postcosecha merecen casi el mismo peso que los enfoques de apoyo a la producción en fincas.

La facilitación del proceso EPCP. En los casos analizados se identifican tres tipos distintos de "campeones", que contribuyen a la facilitación del proceso, que pueden ser cruciales para la implementación exitosa del EPCP y para la integración del enfoque en las organizaciones de I+D. El primer tipo de campeón es el que coordina los grupos de trabajo e interviene en articular los procesos de innovación; el segundo tipo es un gerente / tomador de decisiones que facilita la movilización de recursos para el EPCP, además de coordinar de forma general la implementación del enfoque; el tercer tipo de campeón es un líder reconocido dentro de la cadena de mercado y que tenga buenas conexiones para la incidencia pública y de políticas. En muchos casos, las instituciones de I+D fueron responsables de introducir $y$ facilitar el proceso de implementación de la metodología en el marco de un proyecto. Los facilitadores de EPCP deben tener habilidades de comunicación y manejo de grupos disimiles (por ejemplo, el trato con empresas privadas es fundamental) y estar dispuestos en aprender y trasmitir nuevas habilidades sobre mercadeo agrícola y procesos de innovación.

Las innovaciones surgen con el tiempo y no son siempre programadas. El EPCP desencadena procesos de innovación, que a menudo continúan y evolucionan mucho después de que el EPCP ha terminado formalmente. Las innovaciones de segunda y tercera generación o lo que llamamos las imitaciones creativas suelen ser hasta más importantes que las primeras innovaciones desarrolladas durante el ejercicio EPCP. Pero este proceso requiere una inversión en servicios y mecanismos institucionales que permitan dar seguimiento y apoyar las innovaciones que surgen más allá de la vida de un proyecto específico. La falta de acompañamiento de las innovaciones en cadena puede tornarse en una limitante que reduce los alcances del proceso de innovación.

El contexto de políticas. Parece ser que las políticas en favor del mercado en Perú proporcionaron un entorno más favorable para el uso del EPCP. Asimismo, en los entornos donde la pobreza es muy elevada y las condiciones de producción son adversas debido a los riesgos climáticos y el uso reducido de insumos, existen limitantes severas para la implementación de los enfoques de desarrollo de las cadenas de mercados agrícolas. Condiciones favorables para 
que estos enfoques prosperen, pasan por entender que las políticas deben facilitar la participación y el desarrollo de los actores privados (productores y empresas) y reducir los costos de transacción para que los negocios funcionen. El EPCP busca entre otras cosas, que tres actores claves (productores, empresas y gobierno) puedan articular objetivos y llevarlos a la práctica. Cuando se pueden reunir estas condiciones, con un componente de desarrollo de capacidades locales, se llega a facilitar procesos de innovación exitosos.

Los enfoques holísticos y participativos, como el EPCP, no pueden ser fácilmente "escalados" o "transferidos". ¿Cómo se puede escalar o replicar mejor las iniciativas observadas? Para alcanzar la réplica de enfoques exitosos es importante fomentar la innovación, el aprendizaje y la difusión de experiencias. Hasta la fecha, la inversión en aprendizaje y difusión de experiencias no ha alcanzado un nivel de apoyo similar a la promoción de iniciativas prácticas en el campo.

No existe una "solución única" para los desafíos a los que se enfrentan las cadenas de valor básicas. No existe un factor único de éxito, sino, en cada caso se necesita una combinación de intervenciones a diferentes niveles para abordar los procesos de desarrollo de cadenas de valor. Más bien, se necesita un conjunto de medidas de política, programas de desarrollo y fortalecimiento de capacidades técnicas en los actores de la cadena para facilitar los procesos de innovación y fortalecer enlaces efectivos para promover la participación de pequeños productores en los mercados en expansión. La incidencia en políticas es una variable importante para asegurar que los Estados se articulen en este proceso. El marco de políticas influye en el desarrollo de los negocios y se deben tener mecanismos de visibilidad ante los tomadores de decisiones. En Perú, se involucró directamente al sector público y se pudo contar con mecanismos de apoyo que complementaban la acción privada (de productores y empresas) y esto generó un entorno positivo que sigue hasta la fecha como base para el desarrollo competitivo.

En el futuro, una investigación comparativa de las variables que influyen y determinan la adaptación de metodologías participativas como el EPCP en diferentes contextos permitiría definir mejor las condiciones de réplica, adaptación y escalamiento de dichas metodologías.

\section{Conflictos de intereses}

El presente documento no presenta conflictos de intereses.

\section{Referencias citadas}

Antezana, I., Bernet, T., López, G. y Oros, R. (2008). Enfoque Participativo en Cadenas Productivas (EPCP): Guía para capacitadores. Centro Internacional de la Papa, Lima, Perú. 189-pp.

Bernet, T., Thiele, G. and Zschocke, T. (2006) Participatory Market Chain Approach (PMCA) - User Guide. Lima: International Potato Center (CIP) (http://cipotato.org/publications/pdf/0032 96.pdf).

Devaux, A., Ordinola, M. and Horton, D. (eds) (2011) Innovation for Development: The Papa Andina Experience. International Potato Center (CIP). Lima, Peru: CIP. 314 pp.

Devaux, A., Ordinola, M., Mayanja, S., Campilan, D. and Horton, D. (2013) The Participatory Market Chain Approach: from the Andes to Africa and Asia. Papa 
Andina Innovation Brief 1. International Potato Center, Lima, Peru. 4 pp.

Devaux, A., Torero, M., Horton, D. \& Donovan, J. (Eds). (2016) Innovation for inclusive value chain development: Successes and challenges. International Food Policy Research Institute (IFPRI), Washington, D.C. 529 pp.

Engel, P. and Salomon, M. (2003) Facilitating Innovation for Development: A RAAKS Resource Box. Amsterdam: Royal Tropical Institute (http://www.kit.nl/kit/Publication?item=1 512).

FAO. 2013. Agroindustrias para el desarrollo. Roma (http://www.fao.org/3/a-i3125s.pdf)

Horton, D. and Samanamud, K. (2013) Peru's native potato revolution. Papa Andina Innovation Brief 2. International Potato Center, Lima, Peru. 6 pp.

Maldonado, L., Ordinola, M., Manrique, K., Fonseca, C., Sevilla, M. y Delgado, O. (2011) Estudio de caso: Evaluación de impacto de la intervención del proyecto INCOPA/CAPAC en Andahuaylas. Lima, Perú, CIP.

Montesdeoca, L., Acosta, M., Quishpe, C., Monteros, C., Andrade-Piedra, J., Pavez, I. (2013) Rescatando variedades ancestrales: innovación de las papas nativas en Ecuador. En: "Innovaciones de Impacto, lecciones de la agricultura familiar en América Latina y el Caribe" / Editado por Priscila Enriquez, Hugo Li Pun, San José de Costa Rica. IICA, BID. 224 p.

Montesdeoca, L. (2016) Reporte trianual 2014-2016 de TRIAS - AGROPAPACONPAPA. 14 pp.

Ordinola, M., Devaux, A., Manrique, K., Fonseca, C., Thomann, A. (2009) Generando innovaciones para el desarrollo competitivo de la papa en el Perú. Lima: Centro Internacional de la Papa (CIP).

Ordinola, M., Devaux, A., Manrique K., Fonseca, C. (2013) Innovaciones en la cadena de la papa en el Perú: el valor de la biodiversidad. Leisa, Revista Agroecológica 29 (2): 10-13.

Ordinola, M., Devaux, A., Bernet, T., Manrique, K., Lopez, G., Fonseca, C., Horton, D. (2014) The PMCA and potato market chain innovation in Peru. Papa Andina Innovation Brief 3. International Potato Center, Lima, Peru. 8 pp.

Proexpansión. 2011. Cambios del sector papa en el Perú en la última década: Los aportes del proyecto Innovación y Competitividad de la Papa (INCOPA). Lima, Perú: Centro Internacional de la Papa.

Reardon, T. and Timmer, P. (2012) "The Economics of the Food System Revolution." Annual Review of Resource Economics 4: 225-264.

Reardon, T., Chen, K., Minten, B. and Adriano, L. (2012) The Quiet Revolution in Staple Food Value Chains: Enter the Dragon, the Elephant, and the Tiger. Asian Development Bank and International Food Policy Research Institute, Mandaluyong City, Philippines and Washington, D. C.

Royer A., Bijman, J. and Bitzer, V. (2016) Linking smallholder farmers to high quality food chains: appraising institutional arrangements, in book: Quality and innovation in food chains, Chapter: 2, Publisher: Wageningen Academic Publishers, Editors: J. Bijman and V. Bitzer, pp.33-62.

Thiele, G., Devaux, A., Reinoso, I., Pico, H., Montesdeoca, F., Pumisacho, M., Andrade-Piedra, J., Velasco, C., Flores, P., Esprella, R., Thomann, A., Manrique, 
K. and Horton, D. (2011b) "Multistakeholder platforms for linking small farmers to value chains: evidence from the Andes." Agricultural International Journal of Sustainability 9(3): 1-11.

Tobin, D., Glenna, L. and Devaux, A. (2016) Pro-poor? Inclusion and exclusion in native potato value chains in the central highlands of Peru. Journal of Rural Studies 46: 71-80.

UNIDO (2011) Pro-Poor Value Chain Development: 25 Guiding Questions for Designing and Implementing Agroindustry Projects. United Nations Industrial Development Organization, Vienna, Austria.
Wiggins, S. and Keats, S. (2013) Leaping and Learning: Linking smallholders to markets in Africa. London: Agriculture for Impact, Imperial College and Overseas Development Institute.

Wong, N. y Padilla, M. (2017) Emprendimiento social para la recuperación de la producción agrícola de patatas nativas de los Andes de Ecuador: Caso KIWA chips. En Experiencias de emprendimiento social en Iberoamérica. Editorial Universidad de Almería. Colección: Libros Electrónicos $\mathrm{N}^{0}$ 67. p. 77-81. 